\title{
PÉRDIDA DE DIVERSIDAD FLORÍSTICA ANTE UN GRADIENTE DE INTENSIFICACIÓN DEL SISTEMA AGRÍCOLA DE ROZA-TUMBA-QUEMA: UN ESTUDIO DE CASO EN LA Selva Lacandona, Chiapas, MÉXICO
}

\author{
Susana Ochoa-Gaona ${ }^{1,4}$, Francisco Hernández-VázQuez², \\ Bernardus H. J. De Jong ${ }^{1}$, Francisco D. Gurri-García ${ }^{3}$ \\ ${ }^{1}$ Departamento de Agroecología, El Colegio de la Frontera Sur. Apartado Postal 1042, Admón. de Correos de Tabasco \\ 2000, C.P. 86031, Villahermosa, Tabasco, México. \\ ${ }^{2}$ Instituto Manantlán de Ecología y Conservación de la Biodiversidad, Universidad de Guadalajara. Apartado Postal \\ 64, 48900, Autlán, Jalisco, México. \\ ${ }^{3}$ El Colegio de la Frontera Sur. Unidad Campeche. Calle 10 esq. Calle 61 No. 264, Col. Centro, 24000 Campeche, \\ Campeche, México. \\ ${ }^{4}$ Autor para la correspondencia. Correo-e: sochoa@ecosur.mx
}

\begin{abstract}
Resumen: Se analizó la dinámica del sistema agrícola de roza-tumba-quema (r-t-q) bajo un proceso de alargamiento de los años de cultivo de la parcela y un incremento de los periodos de cultivo, y se determinó bajo qué intensidad y frecuencia de uso los acahuales conservan especies propias de la flora leñosa nativa en la Zona Norte de la Selva Lacandona. Se levantaron 39 inventarios en acahuales de diferentes edades (EA) e historias de uso. Se generó un índice de integridad (II) que combina la intensidad y la frecuencia de uso de las parcelas. Mediante un análisis canónico de correspondencia se evaluó la relación de las especies con EA e II. Se encontró que los acahuales de mayores edades e II funcionan como reservorios de especies nativas propias del bosque maduro. Las tendencias del sistema de r-t-q son de incremento de su intensidad y frecuencia de uso, por lo que la matriz será mas homogénea y de mayor contraste con las áreas forestales, lo cual impedirá la dispersión, el establecimiento y el mantenimiento de la porción de flora nativa propia de estos hábitats, comprometiendo su sobrevivencia.
\end{abstract}

Palabras clave: bosque tropical, flora leñosa, agricultura tradicional, sucesión secundaria, conservación de especies.

\begin{abstract}
We analyzed the effect of intensifying the traditional slash-and-burn system on floristic diversity in order to determine the threshold of frequency and duration of cultivation below which native woody species can be maintained. A total of 39 floristic inventories were conducted in the Northern Region of the Selva Lacandona of secondary vegetation with different ages (AE) and use history. We constructed an integrity index (II) that combines agricultural use intensity (number of years under cultivation) and frequency of slash-and-burn agriculture. We applied a canonical correspondence analysis to group the species according to $\mathrm{AE}$ and II. We found that fallows originated from slash-and-burn systems with low use intensity and low frequency can act as a reservoir of species of mature tropical forests. However, if the intensification trend of slash-and-burn continues toward longer periods of cultivation and higher frequencies of slash-and-burn, this will create a more homogeneous landscape with a very high contrast with the remaining forest patches, which will limit the dispersal, establishment and sustainability of the portion of primary species that could survive under conditions of low-intensive slash-and-burn agriculture.
\end{abstract}

Key words: tropical rain forest, woody flora, traditional agriculture system, species conservation, secondary vegetation.

$\mathbf{E}^{1}$ sistema agrícola tradicional de roza-tumba-quema (r-tq) fue por más de tres mil años la forma dominante de aprovechamiento de los recursos naturales en las zonas tropicales y subtropicales del planeta (Moya-García et al., 2003), siendo un ejemplo de los pocos sistemas sustentables que hubo entre los indígenas mayas (Hernández-X.,
1959; Toledo, 1995; Quintana-Ascencio et al., 1996; Moya-García et al., 2003). El sistema r-t-q, aunque tiene muchas variantes, de manera general incluye la selección del sitio, el aclareo del bosque mediante el corte de arbustos y bejucos, el derribo de los árboles y la quema de los residuos secos producto de estas dos actividades; posterior- 
mente se procede al cultivo de milpa, abandono y crecimiento del acahual (van der Wal, 1999; Giardina et al., 2000). El proceso de quema incrementa a corto plazo la disponibilidad de nutrientes como N, P y otros cationes, aumentando con ello la producción agrícola (Kauffman et al., 1993; Giardina et al., 2000; Wan et al., 2001). En su forma tradicional, la milpa generalmente se mantiene por periodos de 1 a 3 años y consiste en la siembra de maíz, generalmente asociado con frijol, calabaza y otros productos agrícolas complementarios (Hernández-X., 1959; Toledo, 1995; Toledo et al., 1995; Quintana-Ascencio et al., 1996; García-Moya et al., 2003). Tradicionalmente, la vegetación secundaria leñosa o acahual se desarrolla durante un período de descanso de 15 a 20 años mientras se repone la fertilidad del suelo y se restaura la capacidad productiva del sistema (Alemán-Santillán, 1989; Brown y Lugo, 1990; Pool-Novelo y Hernández-X., 1991; Kwesiga, 1994; Toledo, 1995; Toledo et al., 1995; Pool-Novelo, 1997; Emrich et al., 2000; Fox et al., 2000).

Una vez que la parcela se deja descansar se suceden diferentes etapas sucesionales. Primero, la parcela es colonizada por numerosas hierbas y arbustos provenientes del banco de semillas. Uno o dos años después aparecen especies arbóreas pioneras, las cuales a su vez pueden ser gradualmente reemplazadas por especies arbóreas sucesionalmente intermedias provenientes de la lluvia de semillas de la vegetación arbórea remanente que la rodea (GómezPompa y Vázquez-Yanes, 1976; Quintana-Ascencio et al., 1996; Tucker et al., 1998; Guariguata y Ostertag, 2001). Por otra parte, cuando se dejan árboles remanentes de la vegetación original o sus tocones, se favorece la presencia de especies arbóreas de etapas avanzadas desde las primeras fases de la sucesión (Gómez-Pompa y Vázquez-Yanes, 1976; 1985; Vázquez-Yanes y Guevara-Sada, 1985; Quintana-Ascencio et al., 1996; Ochoa-Gaona y González Espinosa, 2000; ITTO, 2002).

En sus fases maduras, los acahuales pueden funcionar como reservorio de algunas especies nativas, e incluso permitir el paso de algunos organismos al mantener la conectividad con los fragmentos de bosque maduro, amortiguando así el efecto de la fragmentación (Lovejoy et al., 1983; Fahrig y Merriam, 1985; Janzen, 1986; Bierregaard et al., 1992; Kellman, 1996). Sin embargo, en la actualidad, mientras que los períodos de cultivo se han alargado, los de descanso se han reducido a pocos años, lo cual limita el desarrollo de vegetación secundaria leñosa y la renovación de nutrientes del suelo, perdiendo así su función en el sistema (Alemán-Santillán, 1989; Parra-Vázquez y MeraOvando, 1989; De Jong y Montoya-Gómez, 1994; PoolNovelo, 1997; Levy-Tacher, 2000; Ochoa-Gaona y González-Espinosa, 2000).

En el área de estudio, el manejo de la parcela bajo el sistema r-t-q es un proceso individual en el cual cada agricultor decide cuándo y dónde cultivar milpa, así como cuánta superficie destina para tal fin, dependiendo de sus necesidades y posibilidades. Por ello, cada parcela presenta una historia propia en cuanto a la fecha de su establecimiento y la longitud tanto de los períodos de cultivo de milpa como de los de descanso. Esto da como resultado variaciones en la intensidad y frecuencia de uso de las parcelas. En esta zona, el incremento poblacional, los bajos precios de la producción agrícola y la falta de alternativas económicas han llevado a los campesinos indígenas a intensificar el sistema agrícola tradicional de r-t-q. Como resultado de esto se han reducido los períodos de descanso de la parcela de tres a siete años, lo cual limita la renovación de nutrientes del suelo y a la larga el desarrollo de comunidades arbóreas secundarias (Alemán-Santillán, 1989; Pa rra-Vázquez y Mera-Ovando, 1989; De Jong y Montoya-Gómez, 1994; Pool-Novelo, 1997; Levy-Tacher, 2000; Ochoa-Gaona y González-Espinosa, 2000).

Partiendo de la premisa de que el acortamiento de los periodos de descanso limita la capacidad de renovación de nutrientes del suelo y que el incremento de la frecuencia de aplicación de fuego afecta al banco de semillas, la hipótesis de trabajo sostiene que la composición florística de la vegetación secundaria está fuertemente influenciada por la intensidad y la frecuencia de uso de las parcelas, lo que produce un efecto variable en el desarrollo del nuevo acahual y de la flora leñosa nativa que en éste se establece. Es de esperar un mayor número de especies propias de bosque nativo en acahuales de más edad derivados de parcelas con bajas intensidad y frecuencia de uso. En este marco, el objetivo fue analizar bajo qué intensidad y frecuencia de uso, los acahuales derivados del sistema r-t-q son capaces de conservar especies de la flora leñosa propias del bosque en la Zona Norte de la Selva Lacandona.

\section{Área de estudio}

El área de estudio forma parte de la Zona Norte de la Selva Lacandona, la cual se localiza en el nordeste del estado de Chiapas. Abarca el municipio de Palenque y el norte del municipio de Ocosingo (Ramírez-Marcial et al., 1996; De Jong et al., 2000; figura 1b). La cubierta vegetal dominante son pastizales inducidos para la ganadería, áreas agrícolas y acahuales en diferentes etapas sucesionales. Apenas una tercera parte de la superficie queda cubierta por remanentes de bosque (De Jong et al., 2000). De éstos, la mayor parte es bosque tropical perennifolio y subperennifolio, y en menor escala bosque de pino-encino, bosque ripario, jimbales y sabanas (Rzedowski, 1978; Pennington y Sarukhán, 1998; De Jong et al., 2000; INE, 2000). El clima es cálido húmedo con un período de sequía de feb rero a marzo (García-Gil y Lugo-Hupb, 1992). La altitud p redominante fluctúa entre 0 y $300 \mathrm{~m}$, pero alcanza hasta $1500 \mathrm{~m}$. La población está conformada por grupos indígenas — tzeltales, choles, lacandones-y mestizos emigran- 
tes de los municipios de Chilón, Sabanilla, Ocosingo, Yajalón y Salto de Agua. Esta región ha sufrido un proceso continuo de destrucción de los recursos naturales para el desarrollo de pastizales para la ganadería extensiva (De Vos, 1992). La superficie más alterada de este territorio se encuentra dentro de una franja de $2 \mathrm{~km}$ alrededor de los asentamientos humanos o a los lados de los caminos (Sader et al., 1994; O’Brien, 1995).

\section{Materiales y métodos}

Para cubrir diferentes historias de uso de las parcelas y mayor variación de la edad de los acahuales, el trabajo se realizó en las comunidades Río Chancalá y El Naranjo, y en el Parque Nacional Palenque, municipio de Palenque, Chiapas (figura 1; cuadro 1).

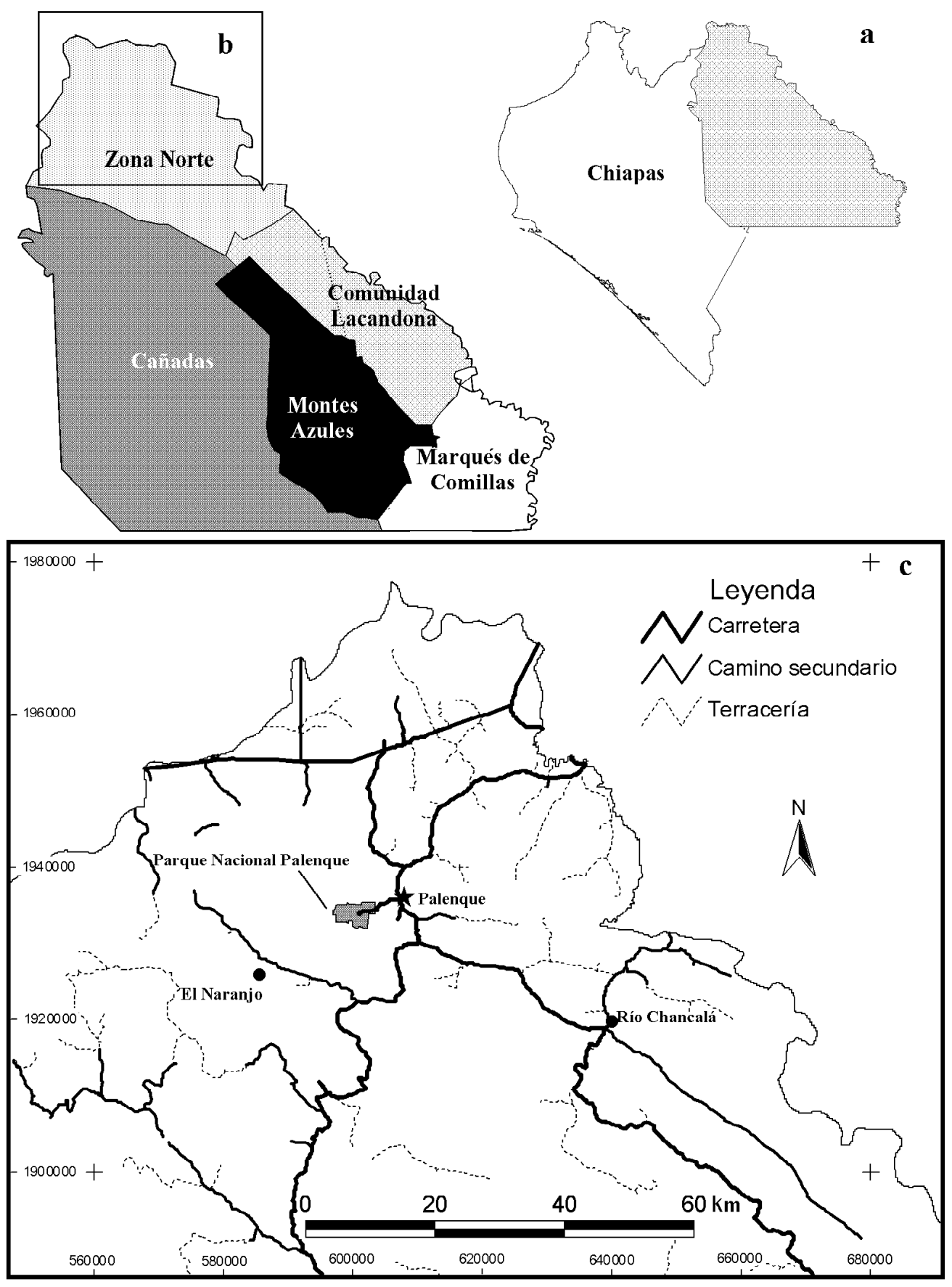

Figura 1. Mapa de localización del área de estudio. (a) Ubicación de la Selva Lacandona en el estado de Chiapas. (b) Ubicación de la Zona Norte y las otras subregiones de la Selva Lacandona (INE, 2000). (c) Ubicación de las comunidades de estudio. 
Cuadro 1. Caracterización de las comunidades de estudio

\begin{tabular}{|c|c|c|c|}
\hline & \multicolumn{3}{|c|}{ Comunidades estudiadas } \\
\hline & Parque Nacional Palenque & El Naranjo & Río Chancalá \\
\hline Categoría & Área natural protegida & Comunidad indígena & Comunidad indígena \\
\hline Decreto de establecimiento & 1981 & 1954 & 1960 \\
\hline Clima & Cálido subhúmedo 22 a $26^{\circ} \mathrm{C}$ & Cálido subhúmedo 22 a $26^{\circ} \mathrm{C}$ & Cálido subhúmedo 22 a $26^{\circ} \mathrm{C}$ \\
\hline Tipos de suelo & $\begin{array}{l}\text { Rendzina, Feozems, } \\
\text { Vertisoles y Fluvisoles }\end{array}$ & Vertisoles y Fluvisoles & Rendzina y Feozems \\
\hline Altitud (m) & 60 a 200 & 269 a 600 & 100 a 200 \\
\hline Vegetación & $\begin{array}{l}\text { Domina selva alta perennifolia } \\
\text { alternada con acahuales y } \\
\text { pastizales inducidos }\end{array}$ & $\begin{array}{l}\text { Dominan los acahuales en } \\
\text { diferentes estados de desarrollo } \\
\text { y áreas de cultivo }\end{array}$ & $\begin{array}{l}\text { Dominan pastizales y } \\
\text { cultivo de milpa bajo r-t-q, } \\
\text { pocos acahuales }\end{array}$ \\
\hline Actividades productivas & Conservación, ganadería y milpa & $\begin{array}{l}\text { Principalmente milpa, también } \\
\text { se produce cacao y café }\end{array}$ & $\begin{array}{l}\text { Ganadería y milpa, también } \\
\text { chile y frijol }\end{array}$ \\
\hline Principales problemas & $\begin{array}{l}\text { Conflictos por tenencia de la } \\
\text { tierra con ganaderos afectados por } \\
\text { el decreto, aún realizan actividades } \\
\text { agrícolas y ganaderas dentro del } \\
\text { parque }\end{array}$ & $\begin{array}{l}\text { Bajos precios del maíz, falta de } \\
\text { caminos y transporte para sacar } \\
\text { sus productos al mercado, baja } \\
\text { productividad del suelo, poco } \\
\text { interés por trabajar la tierra } \\
\text { principalmente de los jóvenes }\end{array}$ & $\begin{array}{l}\text { La gente emigra en busca de } \\
\text { trabajo a la ciudad de Guadalajara } \\
\text { y a los Estados Unidos de } \\
\text { Norteamérica, y renta las } \\
\text { parcelas a ganaderos por lo que } \\
\text { no hay abandono de la tierra para } \\
\text { el desarrollo de acahuales }\end{array}$ \\
\hline
\end{tabular}

Estructura y composición. Para caracterizar la estructura y la composición florística de los acahuales, se realizaron inventarios florísticos. En cada sitio se tomaron datos del número de estratos y de altura del dosel, y con base en los inventarios se calculó el área basal. Asimismo, se identificaron, registraron y midieron las plantas leñosas en un área de muestreo de un círculo de $1,000 \mathrm{~m}^{2}(\mathrm{r}=17.84 \mathrm{~m})$ y dentro de éste se establecieron círculos concéntricos de 500 y $100 \mathrm{~m}^{2}$ ( $\mathrm{r}=12.62 \mathrm{~m}$ y $5.64 \mathrm{~m}$, respectivamente). En los círculos de $1,000 \mathrm{~m}^{2}$ se identificaron, registraron y midieron los árboles con diámetro a la altura del pecho (DAP) $40 \mathrm{~cm}$; en los de $500 \mathrm{~m}^{2}$ se hizo lo mismo a los árboles con DAP $20 \mathrm{~cm}$; y en los de $100 \mathrm{~m}^{2}$ todos los árboles con DAP $5 \mathrm{~cm}$. Los árboles con DAP $<5 \mathrm{~cm}$ pero $>1.30 \mathrm{~m}$ de altura (juveniles) fueron contados, identificados y registrados dentro del círculo de $100 \mathrm{~m}^{2}$. Los árboles con altura $<1.30 \mathrm{~m}$ (plántulas) fueron contados, identificados y registrados en dos cuadros de 4 $\mathrm{m}^{2}$ ubicados en el perímetro del círculo de $1,000 \mathrm{~m}^{2}$. En total se realizaron 39 inventarios florísticos, cada uno con su respectiva historia de uso de la parcela. En el Parque Nacional Palenque se realizaro 19 inventarios y en El Naranjo y Río Chancalá, 10 inventarios en cada localidad.

En cada acahual se recolectaron muestras botánicas de las diferentes especies encontradas. Para cada espécimen se registró su nombre común, forma biológica y características dendrológicas (De Jong et al., 1990; López-García, 1999). La determinación de la especie se realizó mediante la comparación con un catálogo de campo de la Selva
Lacandona, con especímenes del herbario $\mathrm{CH}$ de ECOSUR-SCLC y con el apoyo de taxónomos y parataxónomos. Las especies no identificadas se registraron como morfoespecies.

Las especies se clasificaron como propias de bosques maduros y propias de vegetación secundaria con base en Pennington y Sarukhán (1998). De acuerdo con estos autores, las especies de bosque maduro son las que forman los estratos medio y superior de los bosques tropicales en México; en general, se trata de especies intolerantes a la luz, mientras que las especies propias de vegetación secundaria corresponden a especies tolerantes a la luz y que se desarrollan después de la aplicación del sistema r-t-q, considerando tanto especies de vegetación secundaria joven como de vegetación secundaria avanzada (apéndice 1). Con esta base, las especies se agruparon en cuatro grupos: TEL (total de especies leñosas incluyendo plántulas y juveniles), TEPB (total de especies propias de bosques maduros, incluyendo plántulas y juveniles), EPB (total de especies leñosas propias de bosque $5 \mathrm{~cm} \mathrm{D} \mathrm{AP),} \mathrm{y} \mathrm{EL} \mathrm{(total} \mathrm{de}$ especies leñosas $5 \mathrm{~cm} \mathrm{D} \mathrm{AP).}$

Historia de uso. Para conocer la historia de uso de la parcela y la edad del acahual se aplicaron encuestas estructuradas a los dueños de las mismas. En cada encuesta se registraron las siguientes variables: fecha en que se derribó la vegetación original (años de uso, $\mathrm{AU}$ ); número de intervalos que cultivó milpa en la parcela a lo largo de su histo- 
ria (número de periodos de cultivo, $\mathrm{PC}$ ); longitud de tiempo de cada período en el que la parcela se utilizó de manera continua como milpa (años de cultivo, AC); número de veces que se ha iniciado el desarrollo de un acahual a lo largo de la historia de uso de la parcela (orden del acahual, OA); duración de cada período de descanso en el cual se desarrolló el acahual para la recuperación de la fertilidad de suelo (años de descanso, AD); tiempo de descanso del acahual evaluado en este estudio, el cual se desarrolló después del último período de cultivo de la parcela (edad del acahual, EA), y longitud de tiempo de cada período en el que la parcela se utilizó de manera continua como pastizal inducido (años de pastizal, AP).
Índice de integridad. Para relacionar los factores de manejo de las parcelas se propuso un índice de integridad (II) adaptado del índice de disturbio de Levy-Tacher (2000). En este índice, se incluyen la duración de los períodos de descanso (AD), la duración de los períodos de cultivo de milpa (AC) y la duración de los períodos de pastizal para la ganadería (AP), siendo interpretado el efecto de estas variables como la intensidad de uso de las parcelas (Iu; cuadro 2). El número de periodos de cultivo (PC) es usado como indicador de la frecuencia de uso de la parcela. Las fórmulas que se utilizaron fueron: (a) para parcelas que en su historia solo hubo alternancia milpa-acahual, y (b) para parcelas que presentaron períodos de pastizal para la ganadería.

Cuadro 2. Historia de manejo de las 39 parcelas. $A D=$ años de descanso de los acahuales en períodos previos al actual, $A C=$ años de cultivo por periodo, $\mathrm{AP}=$ años de pastizal, $\mathrm{AU}=$ años de uso, $\mathrm{EA}=$ edad del acahual, lu = intensidad de uso, II = índice de integridad, $\mathrm{OA}=$ orden del acahual, PC = períodos de cultivo (ver la definición de los conceptos y abreviaturas en métodos - historia de uso).

\begin{tabular}{|c|c|c|c|c|c|c|c|c|c|c|}
\hline ID & Comunidad & $\mathrm{AU}$ & PC & $\mathrm{AC}$ & AP & $\mathrm{AD}$ & OA & EA & lu* & $\mathrm{II}^{* *}$ \\
\hline 1 & Río Chancalá & 40 & 11 & 2 & 0 & 1.5 & 11 & 3 & 0.8 & 0.07 \\
\hline 2 & Río Chancalá & 37 & 1 & 3 & 30 & 4 & 1 & 4 & 0.1 & 0.08 \\
\hline 3 & Río Chancalá & 41 & 7 & 3 & 0 & 3 & 7 & 2 & 1.0 & 0.14 \\
\hline 4 & Río Chancalá & 40 & 9 & 2 & 0 & 2.5 & 9 & 2 & 1.3 & 0.14 \\
\hline 5 & Río Chancalá & 37 & 6 & 3 & 0 & 2.5 & 6 & 6 & 0.8 & 0.17 \\
\hline 6 & Río Chancalá & 29 & 9 & 1 & 0 & 2 & 9 & 4 & 2.0 & 0.25 \\
\hline 7 & Río Chancalá & 20 & 3 & 3 & 0 & 3 & 3 & 5 & 1.0 & 0.41 \\
\hline 8 & Río Chancalá & 42 & 5 & 1.2 & 0 & 7.3 & 5 & 7 & 6.1 & 1.21 \\
\hline 9 & Río Chancalá & 42 & 5 & 1 & 0 & 7.3 & 5 & 8 & 7.3 & 1.49 \\
\hline 10 & Río Chancalá & 20 & 3 & 1 & 0 & 5.5 & 3 & 6 & 5.5 & 1.89 \\
\hline 11 & El Naranjo & 29 & 5 & 1 & 0 & 4 & 5 & 8 & 4.0 & 0.96 \\
\hline 12 & El Naranjo & 26 & 4 & 1 & 0 & 7 & 4 & 1 & 7.0 & 1.38 \\
\hline 13 & El Naranjo & 33 & 4 & 1 & 0 & 8.3 & 4 & 4 & 8.3 & 1.81 \\
\hline 14 & El Naranjo & 25 & 3 & 1 & 0 & 4 & 3 & 14 & 4.0 & 2.44 \\
\hline 15 & El Naranjo & 25 & 3 & 1 & 0 & 7 & 3 & 8 & 7.0 & 2.44 \\
\hline 16 & El Naranjo & 31 & 3 & 1 & 0 & 12 & 3 & 4 & 12.0 & 3.11 \\
\hline 17 & El Naranjo & 26 & 2 & 1.5 & 0 & 7 & 2 & 16 & 4.7 & 3.83 \\
\hline 18 & El Naranjo & 5 & 1 & 1 & 0 & 4 & 1 & 4 & 4.0 & 4.00 \\
\hline 19 & El Naranjo & 38 & 2 & 2 & 0 & 4 & 2 & 30 & 2.0 & 4.25 \\
\hline 20 & El Naranjo & 8 & 1 & 1 & 0 & 7 & 1 & 7 & 7.0 & 7.00 \\
\hline 21 & Parque Nacional Palenque & 45 & 4 & 5 & 11 & 3.3 & 4 & 9 & 0.2 & 0.15 \\
\hline 22 & Parque Nacional Palenque & 45 & 4 & 3.8 & 9 & 4 & 4 & 9 & 0.2 & 0.18 \\
\hline 23 & Parque Nacional Palenque & 40 & 3 & 3 & 13 & 3 & 3 & 12 & 0.1 & 0.21 \\
\hline 24 & Parque Nacional Palenque & 46 & 3 & 8 & 0 & 5 & 3 & 12 & 0.6 & 0.31 \\
\hline 25 & Parque Nacional Palenque & 40 & 3 & 4 & 10 & 4 & 3 & 14 & 0.2 & 0.32 \\
\hline 26 & Parque Nacional Palenque & 35 & 2 & 2 & 9 & 3 & 2 & 19 & 0.2 & 0.63 \\
\hline 27 & Parque Nacional Palenque & 47.8 & 4 & 3 & 3 & 4.6 & 4 & 22 & 0.6 & 0.66 \\
\hline 28 & Parque Nacional Palenque & 48 & 4 & 2 & 0 & 5 & 4 & 25 & 2.5 & 1.25 \\
\hline 29 & Parque Nacional Palenque & 49 & 16 & 2 & 0 & 1 & 16 & 2 & 0.5 & 0.03 \\
\hline 30 & Parque Nacional Palenque & 30 & 15 & 1 & 0 & 1 & 15 & 1 & 1.0 & 0.07 \\
\hline 31 & Parque Nacional Palenque & 36 & 12 & 1 & 0 & 2 & 12 & 2 & 2.0 & 0.17 \\
\hline 32 & Parque Nacional Palenque & 40 & 2 & 3 & 17 & 16 & 2 & 1 & 0.6 & 0.27 \\
\hline 33 & Parque Nacional Palenque & 40 & 2 & 2 & 18 & 2 & 2 & 16 & 0.1 & 0.29 \\
\hline 34 & Parque Nacional Palenque & 38 & 4 & 1 & 10 & 7.3 & 4 & 2 & 0.5 & 0.31 \\
\hline 35 & Parque Nacional Palenque & 41 & 6 & 2 & 0 & 2.5 & 6 & 16 & 1.3 & 0.40 \\
\hline 36 & Parque Nacional Palenque & 51 & 10 & 1 & 0 & 2.5 & 10 & 18 & 2.5 & 0.41 \\
\hline 37 & Parque Nacional Palenque & 11 & 3 & 1.6 & 0 & 2 & 3 & 2 & 1.3 & 0.42 \\
\hline 38 & Parque Nacional Palenque & 30 & 3 & 3 & 0 & 3 & 3 & 15 & 1.0 & 0.78 \\
\hline 39 & Parque Nacional Palenque & 25 & 2 & 3 & 2 & 19 & 2 & 1 & 3.2 & 1.67 \\
\hline
\end{tabular}

* Valores más bajos de lu, mayor intensidad de uso de la parcela

** Valores más altos de II, mayor integridad de la parcela 
Cuando un acahual tuvo un uso previo de pastizal para la producción ganadera, al número de años de pastizal (AP) se le multiplicó por un factor de 1.5 , ya que se consideró que hay mayor intensidad de uso, lo que reduce la capacidad de crecimiento del acahual.

$$
\text { (a) } I I=\left(\frac{A D}{A C}\right) / P C
$$

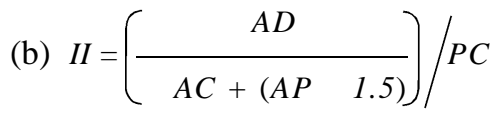

Los valores de II >1 indican mayor integridad, ya que se trata de parcelas con baja intensidad y/o frecuencia de uso.
Estas parcelas pudieron haber tenido periodos de descanso más largos que los periodos de cultivo, o presentaron pocos periodos de cultivo a lo largo de su historia, o ambas posibilidades.

Análisis estadísticos. Se realizaron análisis de correlación entre: (a) las variables número de estratos, altura del dosel y área basal con la edad del acahual para evaluar su posible relación, y (b) para evaluar la independencia entre el índice de integridad (II), la edad del acahual (EA) y el tiempo de uso de la parcela (AU). Se aplicaron análisis de regresión lineal simple para evaluar la relación entre los grupos de especies TEL, TEPB, EPB y EL (cuadro 3) con la edad del acahual, el índice de integridad y los años de uso.

Cuadro 3. Características físicas y estructurales de los 39 acahuales. $A=$ Altitud $(\mathrm{m}) . \mathrm{T}=$ Topografía: $l a=$ ladera, on $=$ ondulada, $\mathrm{pi}=\mathrm{pie}$ de ladera, $\mathrm{pl}=$ plana. $\mathrm{ALD}=$ altura del dosel $(\mathrm{m})$. $\mathrm{NE}=$ número total de estratos. $\mathrm{AB}=$ área basal $\left(\mathrm{m}^{2} / \mathrm{ha}\right) . \mathrm{TEL}=$ número total de especias leñosas incluyendo juveniles y plántulas. EL = número total de especies leñosas $5 \mathrm{~cm}$ DAP. TEPB = número total de especies leñosas propias de bosques maduros incluyendo juveniles y plántulas. $\mathrm{EPB}=$ número de especies leñosas propias de bosque $5 \mathrm{~cm}$ DAP.

\begin{tabular}{|c|c|c|c|c|c|c|c|c|c|c|}
\hline ID & Comunidad & A & $\mathrm{T}$ & ALD & $\mathrm{NE}$ & $\mathrm{AB}$ & TEL & EL & TEPB & $\mathrm{EPB}$ \\
\hline 1 & Río Chancalá & 150 & $\mathrm{pl}$ & 2.1 & 3 & 0.36 & 9 & 1 & 4 & 0 \\
\hline 2 & Río Chancalá & 170 & la & 3.8 & 4 & 9.94 & 12 & 7 & 5 & 3 \\
\hline 3 & Río Chancalá & 100 & pi & 3.6 & 3 & 9.98 & 11 & 3 & 7 & 1 \\
\hline 4 & Río Chancalá & 150 & $\mathrm{pl}$ & 4 & 4 & 10.18 & 13 & 5 & 7 & 1 \\
\hline 5 & Río Chancalá & 150 & la & 4.5 & 4 & 6.23 & 11 & 4 & 6 & 1 \\
\hline 6 & Río Chancalá & 150 & on & 4 & 3 & 6.63 & 9 & 2 & 3 & 1 \\
\hline 7 & Río Chancalá & 160 & pi & 4.5 & 4 & 11.03 & 17 & 5 & 11 & 3 \\
\hline 8 & Río Chancalá & 160 & pi & 5.8 & 3 & 8.35 & 9 & 5 & 3 & 1 \\
\hline 9 & Río Chancalá & 120 & $\mathrm{pl}$ & 7.1 & 3 & 31.79 & 7 & 5 & 4 & 1 \\
\hline 10 & Río Chancalá & 200 & la & 3.8 & 3 & 1.86 & 14 & 6 & 8 & 3 \\
\hline 11 & El Naranjo & 297 & la & 8.1 & 4 & 17.68 & 7 & 4 & 4 & 1 \\
\hline 12 & El Naranjo & 572 & la & 2 & 2 & 0 & 7 & 0 & 3 & 0 \\
\hline 13 & El Naranjo & 512 & la & 3.1 & 4 & 3.47 & 8 & 5 & 5 & 3 \\
\hline 14 & El Naranjo & 421 & la & 11.7 & 5 & 36.32 & 8 & 3 & 6 & 2 \\
\hline 15 & El Naranjo & 380 & la & 10.5 & 4 & 34.81 & 9 & 3 & 4 & 1 \\
\hline 16 & El Naranjo & 540 & la & 6 & 4 & 7.56 & 9 & 7 & 4 & 3 \\
\hline 17 & El Naranjo & 543 & la & 11.5 & 4 & 21.97 & 14 & 7 & 7 & 3 \\
\hline 18 & El Naranjo & 269 & la & 8.5 & 4 & 11.04 & 16 & 8 & 10 & 2 \\
\hline 19 & El Naranjo & 400 & on & 15.1 & 5 & 29.23 & 21 & 13 & 18 & 10 \\
\hline 20 & El Naranjo & 600 & la & 9.5 & 5 & 25.60 & 18 & 9 & 13 & 3 \\
\hline 21 & Parque Nacional Palenque & 60 & $\mathrm{pl}$ & 13.5 & 5 & 35.45 & 16 & 9 & 8 & 4 \\
\hline 22 & Parque Nacional Palenque & 160 & on & 19 & 5 & 24.84 & 14 & 5 & 7 & 1 \\
\hline 23 & Parque Nacional Palenque & 160 & pi & 17.5 & 5 & 25.02 & 20 & 7 & 15 & 6 \\
\hline 24 & Parque Nacional Palenque & 90 & la & 16.5 & 5 & 20.38 & 20 & 8 & 15 & 7 \\
\hline 25 & Parque Nacional Palenque & 195 & pi & 22.5 & 5 & 29.81 & 17 & 7 & 9 & 3 \\
\hline 26 & Parque Nacional Palenque & 199 & $\mathrm{pl}$ & 9 & 5 & 14.32 & 20 & 8 & 16 & 6 \\
\hline 27 & Parque Nacional Palenque & 100 & la & 11 & 5 & 20.48 & 19 & 10 & 12 & 5 \\
\hline 28 & Parque Nacional Palenque & 139 & la & 11 & 4 & 41.43 & 20 & 9 & 13 & 5 \\
\hline 29 & Parque Nacional Palenque & 185 & on & 6.5 & 4 & 9.74 & 15 & 5 & 5 & 1 \\
\hline 30 & Parque Nacional Palenque & 83 & $\mathrm{pl}$ & 2.3 & 2 & 0 & 15 & 1 & 7 & 0 \\
\hline 31 & Parque Nacional Palenque & 75 & on & 2.4 & 3 & 6.75 & 12 & 2 & 6 & 1 \\
\hline 32 & Parque Nacional Palenque & 164 & pi & 1.8 & 3 & 13.66 & 16 & 5 & 7 & 1 \\
\hline 33 & Parque Nacional Palenque & 146 & la & 10 & 5 & 33.97 & 29 & 12 & 18 & 6 \\
\hline 34 & Parque Nacional Palenque & 120 & $\mathrm{pl}$ & 4.5 & 3 & 12.27 & 17 & 6 & 7 & 0 \\
\hline 35 & Parque Nacional Palenque & 100 & on & 10.8 & 5 & 35.46 & 19 & 8 & 13 & 4 \\
\hline 36 & Parque Nacional Palenque & 193 & $\mathrm{pl}$ & 9.5 & 5 & 33.96 & 13 & 6 & 7 & 2 \\
\hline 37 & Parque Nacional Palenque & 73 & $\mathrm{pl}$ & 3 & 3 & 5.15 & 16 & 3 & 10 & 1 \\
\hline 38 & Parque Nacional Palenque & 111 & la & 10.8 & 5 & 18.55 & 20 & 8 & 12 & 3 \\
\hline 39 & Parque Nacional Palenque & 100 & la & 2.3 & 3 & 3.97 & 12 & 3 & 8 & 1 \\
\hline
\end{tabular}


Se realizó un análisis canónico de correspondencia (ACC) para evaluar la relación entre la composición del total de especies leñosas (TEL) con II, EA y AU. El ACC se aplicó sin transformar los datos y sin ponderar ni a las especies ni a los acahuales. Mediante la prueba de Monte Carlo se determinó si los valores propios de los dos primeros ejes de la ordenación, los valores de correlación entre las especies y las variables de manejo eran estadísticamente significativos. Se hicieron 1,000 permutaciones sin restricciones bajo un modelo reducido.

\section{Resultados}

Composición florística. Se registró un total de 119 especies leñosas pertenecientes a 42 familias botánicas. De éstas, 60 especies fueron consideradas nativas propias de bosque maduro. La familia Leguminosae (que incluye Mimosaceae, Caesalpiniaceae y Fabaceae) fue la más abundante en cuanto al número de especies (22), seguida por Rubiaceae (9), Moraceae (6) y Euphorbiaceae (6). Se encontraron entre 7 y 29 especies leñosas por parcela, de las cuales 3 a 18 fueron propias de bosque maduro (cuadro 3 ). Cuarenta y ocho especies fueron registradas exclusivamente en un estado de desarrollo, ya sea como adultos (8), juveniles (31) o plántulas (9). Treinta y cuatro especies se encontraron en los tres estados de desarrollo. Veinte especies se registraron como adultos y juveniles, 16 como juveniles y plántulas, y sólo una especie se registró como adulto y plántula (apéndice 1).

Dinámica de uso de las parcelas. La estrategia de producción de los campesinos dueños de las 39 parcelas estudiadas siguió tres rutas diferentes: $74 \%$ mantuvo la dinámica tradicional de milpa-acahual-milpa, $15 \%$ pasó de milpa a pastizal para la ganadería y $10 \%$ cambió de acahual a pastizal inducido (figura 2). Veintitrés por ciento del total de acahuales presentó edades de 15 años, los cuales en principio se consideran en fase de desarrollo hacia bosque secundario o acahual arbóreo.

Historia de uso de las parcelas. Casi tres cuartas partes (72\%) de las parcelas tuvieron 30 años de uso, 20\% entre 20 y 30 años de uso y sólo $8 \%$ fueron parcelas recientemente incorporadas al sistema r-t-q, con menos de 10 años de uso (el promedio del número de años de uso y el intervalo de confianza del $95 \%$ fueron de $34.1 \pm 3.44$, respectivamente). El número de periodos de cultivo (PC) no estuvo correlacionado con los años de uso de las parcelas, lo cual indica que no hay un patrón de frecuencia de uso $(R=0.353, P<$ $0.05)$. El número de años en que una parcela es cultivada de manera continua $(\mathrm{AC})$ va rió de 1 a $8(2.1 \pm 0.44)$. El tiempo de descanso (AD) de la parcela varió de 1 a 19 años, aunque en $77 \%$ de las parcelas los períodos de descanso variaron de 2 a 7 años (4.95 \pm 1.17 ; figura 3 , cuadro 2$)$.

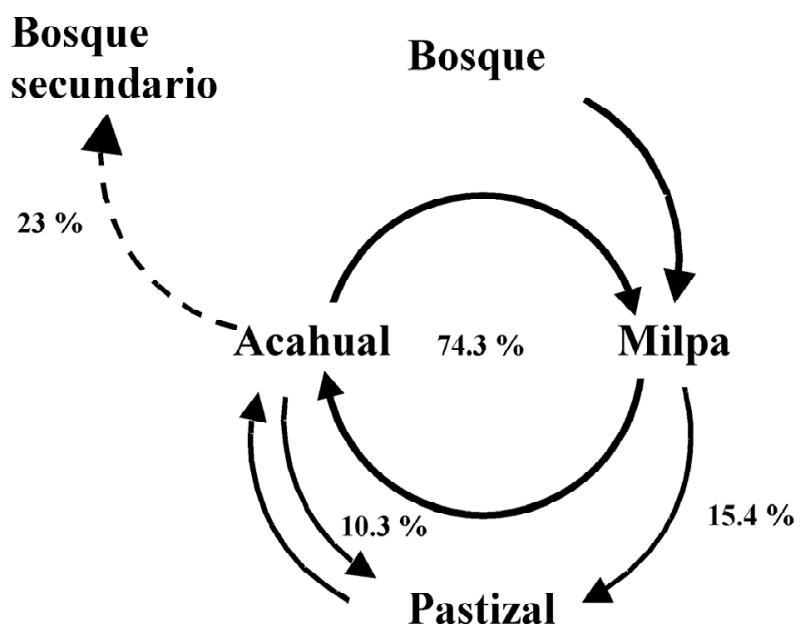

Figura 2. Dinámica de las parcelas en la zona de estudio. Los porcentajes están dados con relación al total de los 39 acahuales. El porcentaje de la flecha punteada corresponde al número de acahuales de 15 años de edad .

En las tres comunidades se encontraron acahuales de 1 a 2 años de edad. La edad máxima varió entre comunidades, siendo el valor máximo de 30 años en El Naranjo (8.74 \pm 2.28; figura 4). En Río Chancalá el paisaje está dominado por pastos para la ganadería que rodean a los acahuales remanentes, los cuales tuvieron edades de 2 a 8 años. En El Naranjo, $60 \%$ de los acahuales presentaron edades de 4 a 8 años, mientras que en el Parque Nacional Palenque 53\% de los acahuales tuvieron de 12 a 25 años de edad, lo cual se debe a que ésta es un área protegida y por ello las actividades de r-t-q se limitaron desde su decreto como Parque Nacional en 1981.

Índice de integridad. El II va rió de 0.033 a 7 (1.169 \pm 0.46$)$. Aunque en promedio el II fue $>1$, solamente $36 \%$ de las parcelas tuvo valores $>1$, mostrando mayor integridad, es decir, parcelas con bajas intensidad y/o frecuencia de uso.

Se encontró una correlación entre el número de estratos, la altura del dosel y el área basal con la edad del acahual ( $R=0.6911,0.6722,0.7158$, respectivamente, todos con $P$ $<0.05)$. Por ello, se tomó la edad del acahual como una variable representativa de la estructura de los acahuales. Los diferentes grupos de especies (cuadro 3) mostraron correlaciones significativas con la edad del acahual $(R=$ $0.5305,0.6494,0.7170,0.7787$, respectivamente, para TEL, TEPB, EL y EPB; $P<0.001$ en todos los casos). Ningún grupo de especies leñosas estuvo correlacionado significativamente con II. La vari able años de uso de la parcela (AU) estuvo negativamente correlacionada con II ( $R=-0.5389, P<0.001)$ y positivamente con la edad del acahual $(R=0.4020, P<0.05)$; por ello AU fue incluida como una covari able en el análisis del ACC. No se encontró una correlación significativa entre II y EA $(R=$ 


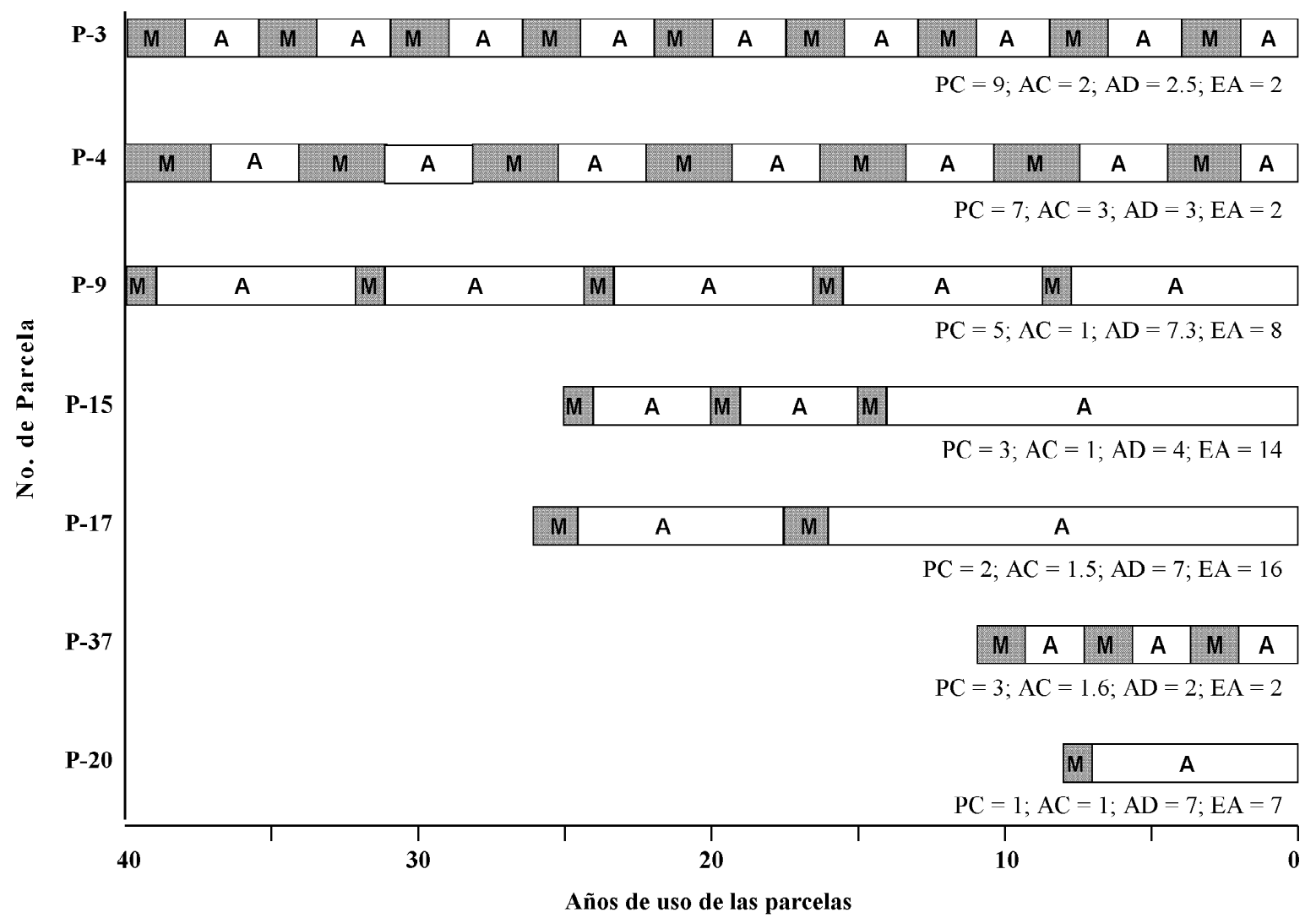

Figura 3. Historia de uso de las parcelas bajo el sistema de roza-tumba-quema. Ejemplos de las parcelas 3, 4, 9, 15, 17, 37 y 20. $\mathrm{M}=$ milpa, $\mathrm{A}=$ acahual $\mathrm{PC}=$ periodos de cultivo, $\mathrm{AC}=$ años de cultivo como milpa, $\mathrm{AD}=$ años de descanso del acahual, $\mathrm{EA}=$ edad actual del acahual. La longitud total del rectángulo indica los años de uso de la parcela (AU; ver definiciones en métodos - historia de uso).

$0.18957, P=0.2477$ ), lo que indica que son variables independientes.

Análisis Canónico de Correspondencia. El ACC reveló que los dos primeros ejes explican $8.5 \%$ de la varianza total de las especies. El eje 1 explicó $4.7 \%$ y se correlacionó positivamente con la edad del acahual $(R=0.937$; cuadro 4$)$. El eje 2 se correlacionó con II $(R=0.849)$, mostrando así la relación de la composición de especies con la intensidad y la frecuencia de uso de las parcelas. La prueba de MonteCarlo mostró una relación estadísticamente significativa $(P$ $<0.001$ ), lo cual indica que las variables II y EA pueden ser utilizadas para explicar la composición de especies de los acahuales. En el diagrama de ordenación sólo se muestran las especies que presentaron un ajuste de $7 \%$ (Ter Braak y Smilauer, 1998). Los acahuales 19 y 20 no fueron incluidos en el análisis dado que por sus valores extremos ejercían una fuerte influencia en la ordenación.

La ordenación ubicó a los acahuales de mayor edad (12 a 25 años) en el lado derecho y a los acahuales de mayor índice de integridad (1.2 a 4.25) en la parte superior de la grafica (figura 5). Las especies que estuvieron más fuerte-

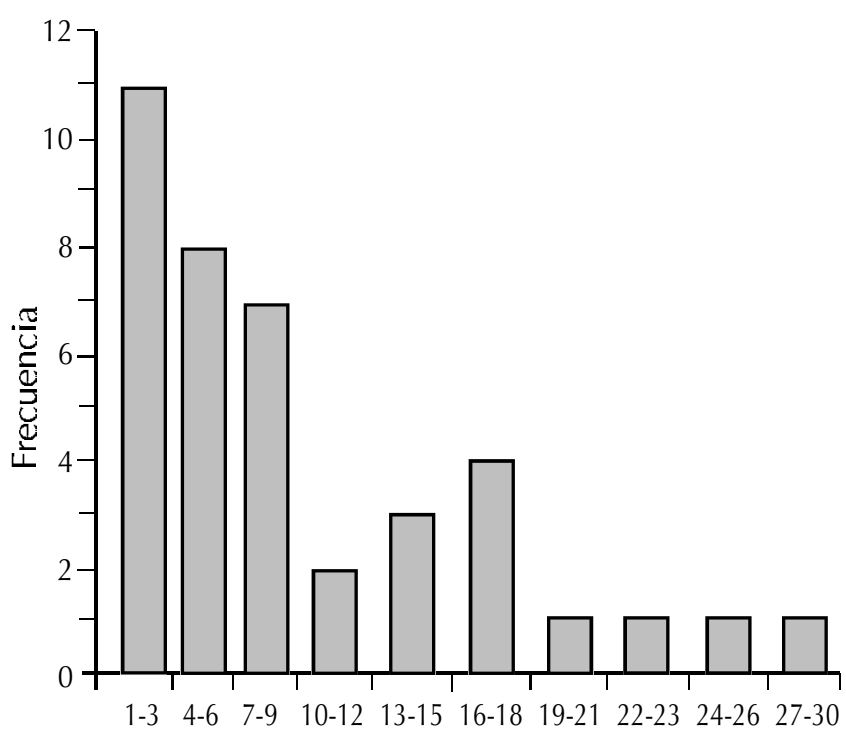

Clases de edad del acahual (años)

Figura 4. Número de acahuales por clase de edad. 


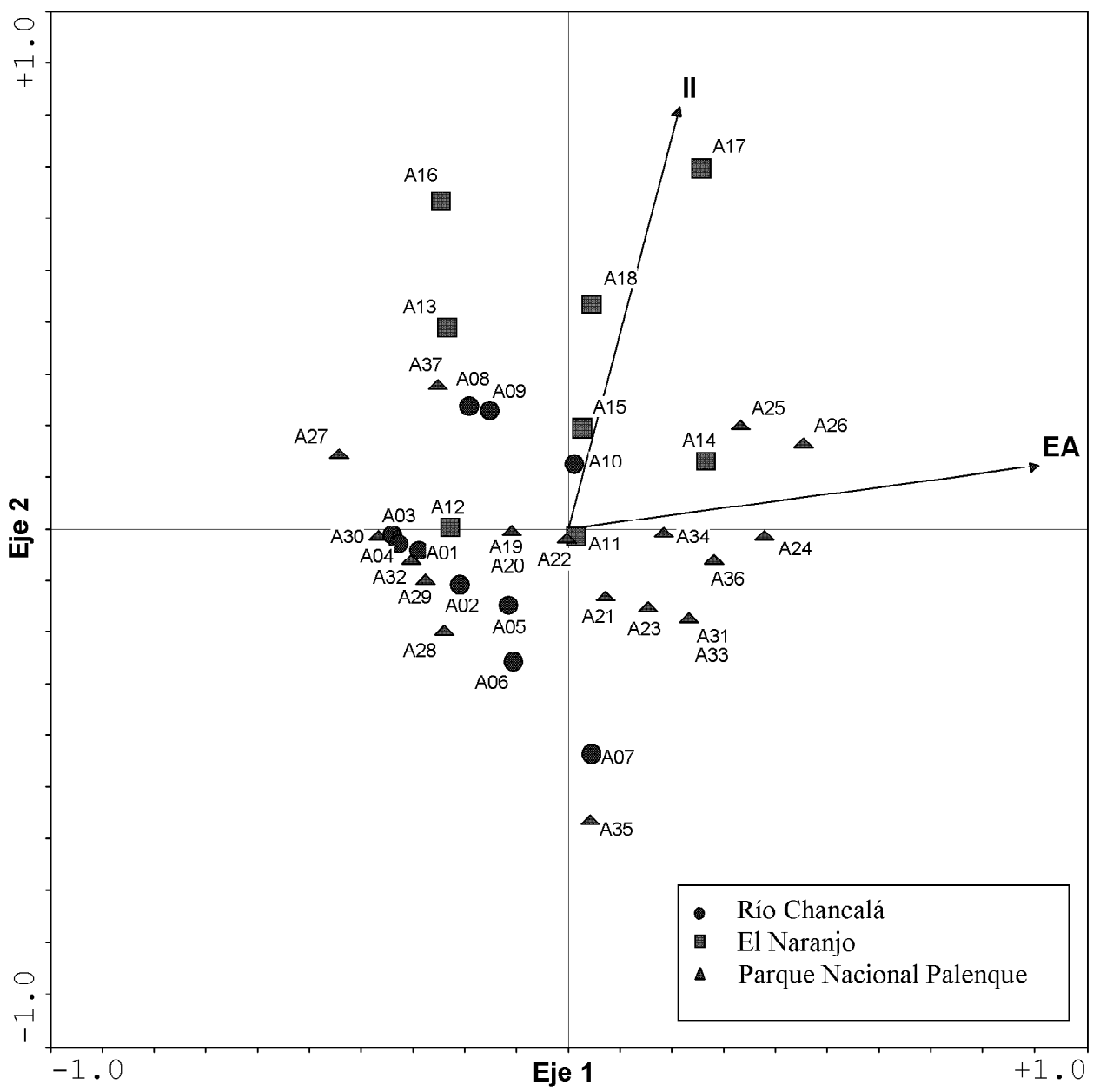

Figura 5. Diagrama de ordenación ACC, mostrando la relación de los acahuales con la edad del acahual (EA) y el índice de integridad (II). Ver cuadro 2 para la correspondencia del número de los acahuales en el diagrama.

Cuadro 4. Resultados del análisis con ACC basado en la presencia / ausencia del total de especies leñosas incluyendo plántulas, juveniles y adultos de las especies propias de bosque maduro y especie de vegetación secundaria.

\begin{tabular}{|c|c|c|c|c|c|}
\hline & \multicolumn{4}{|c|}{ Ejes } & \multirow[b]{2}{*}{ Varianza total } \\
\hline & 1 & 2 & 3 & 4 & \\
\hline Valores propios: & 0.313 & 0.251 & 0.444 & 0.388 & 6.895 \\
\hline $\begin{array}{l}\text { Correlación } \\
\text { especies-variables: }\end{array}$ & 0.946 & 0.879 & 0 & 0 & \\
\hline $\begin{array}{l}\text { Porcentaje de } \\
\text { varianza acumulada } \\
\text { de los datos de } \\
\text { especies: }\end{array}$ & 4.7 & 8.5 & 15.1 & 21 & \\
\hline $\begin{array}{l}\text { de la relación } \\
\text { especies-variables: }\end{array}$ & 55.5 & 100 & 0 & 0 & \\
\hline $\begin{array}{l}\text { Correlación de las } \\
\text { variables en cada eje } \\
\text { Edad del acahual: }\end{array}$ & 0.937 & 0.116 & & & \\
\hline Índice de integridad: & 0.243 & 0.849 & & & \\
\hline
\end{tabular}

mente ligadas a sistemas de bajas intensidad y frecuencia de uso (parcelas con II alto, de 2.4 a 4.25) fueron Coccoloba floribunda, Chrysophylum mexicanum, Erythrina folkersii, Lonchocarpus castilloi, Miconia impe tiolaris y Swartzia cubensis, considerdas propias de bosque maduro, yHeliocarpus popayanensis, Psychotria limo nensis y Trema micrantha, típicas de vegetación secundaria.

Las especies que respondieron más claramente a la edad del acahual fueron Alseis yucatanensis, Blepharidium gua temalense, Ficus yaponensis, Inga pavoniana, Nectandra leucocome, Pseudolmedia oxyphyllaria, Rinorea guatema lensis, Terminalia amazonia y Trichilia montana, que son propias de bosque maduro, y Coccoloba barbadensis, Picramnia aff. teapensis, Rondeletia gonzaleoides y Siparuna andina, que son características de vegetación secundaria.

Las especies más fuertemente ligadas a sistemas de altas intensidad y frecuencia de uso (parcelas con II bajo de 0.033 a 0.96 y edades de uno a nueve años) fueron Acalypha diversifolia, Cestrum nocturnum, Cupania 


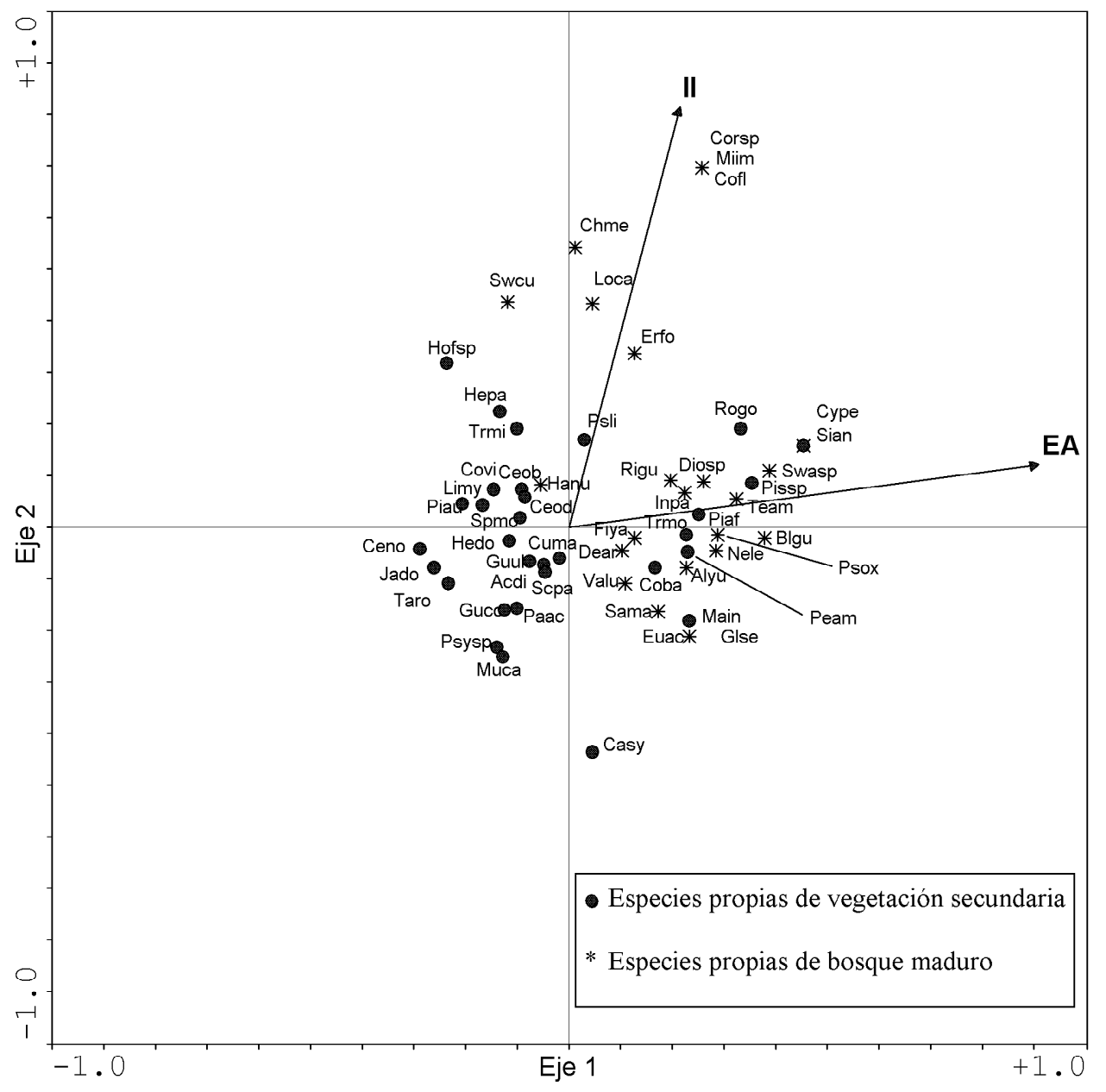

Figura 6. Diagrama de ordenación ACC, mostrando la relación de las especies con la edad del acahual (EA) y el índice de integridad (II). Sólo se muestran las especies con un ajuste 7\%. Ver apéndice 1 para los acrónimos de las especies.

macrophylla, Guazuma ulmifolia, Guettarda combsii, Heliocarpus donnell-smithii, Jacaratia dolichaula, Muntingia calabura, Parmentiera aculeata, Schizolobium parahyba, Spondias mombin y Tabebuia rosea, todas de vegetación secundaria joven (figura 6; cuadro 2).

\section{Discusión}

Paisaje y el sistema r-t-q. La Zona Norte de la Selva Lacandona refleja un proceso histórico de cambio de uso del suelo similar al de otras regiones tropicales (Brown y Lugo, 1990; Dirzo y García, 1992; De Jong et al., 2000; Lambin et al., 2001). El bosque maduro fue parcialmente convertido a tierras de cultivo alrededor de los años cincuentas, y posteriormente, en los años setentas, los remanentes de bosques y parte de las áreas de cultivo se convirtieron en pastizales para la ganadería. Estos procesos de conversión están entre los principales factores responsables de la deforestación en los trópicos (Dirzo y García, 1992;
Fox et al., 2000; Lambin et al., 2001; Carr, 2004; Alvarado et al., 2004). El sistema r-t-q constituye una forma de uso del suelo secuencial e iterativo (van der Wal, 1999), consistente en un mosaico de áreas de cultivo y acahuales en diferentes estados de desarrollo, en el cual el paisaje no queda desprovisto de vegetación, lo que favorece un sistema productivo más o menos sustentable en términos de su impacto sobre los bosques tropicales y su biodiversidad (Smith et al., 1997; Gómez-Pompa y Kaus, 1999).

Dado que los remanentes de bosque maduro están rodeados por una matriz de vegetación secundaria en diferentes etapas sucesionales (Uhl, 1987; De Jong et al., 2000; Ochoa-Gaona y González-Espinosa, 2000; Ochoa-Gaona, 2001), se posibilita la dispersión de al menos una parte de las especies propias del bosque tropical, ya que en los acahuales se genera una estructura básica - de cobertura y altura de la vegetación- que facilita la colonización, el establecimiento de plántulas y el desarrollo de juveniles de las especies leñosas nativas más tolerantes a la sombra (Uhl 
et al., 1981; Brown y Lugo, 1990; Quintana-Ascencio et al., 1996; van der Wal, 1999; Levy-Tacher, 2000; Guariguata y Ostertag, 2001; Carnevale y Montagnini, 2002; Freemark et al., 2002; Chinea y Helmer, 2003).

En nuestro estudio, la mayoría de las especies propias del bosque maduro que se encontraron en los acahuales, como Alseis yucatanensis, Blepharidium guatemalense, Brosimum alicastrum, Cymbopetalum penduliflorum, Dialium guianense, Platymiscium yucatanum, Poulsenia armata, Pseudolmedia oxyphyllaria, Quararibea funebris, Swietenia macrophylla y Terminalia amazonia, fueron registradas sólo como plántulas, como juveniles, o en ambos estadios. Esto indica que estas especies se están estableciendo una vez que los acahuales alcanzan cierto grado de complejidad estructural, ligada en este caso con la edad del acahual. Esto revela una interacción natural entre el bosque maduro y los acahuales, ya que la presencia cercana de bosque maduro, cada vez más escaso, define en parte la sucesión desde las primeras etapas (Gómez-Pompa y Kaus, 1999), ya que no se cuenta con un banco de semillas de especies propias de bosque maduro (QuintanaAscencio et al., 1996). Por ello, dado el progresivo proceso de pérdida de bosques maduros y debido a que el actual sistema r-t-q está acortando el tiempo de desarrollo de la vegetación secundaria, el mantenimiento de esta interacción natural queda cada vez más restringida, afectando los mecanismos de preservación de especies nativas y la pérdida de sustentabilidad de los sistemas tradicionales de producción.

El área de estudio no es la única que mantiene esta dinámica de mosaicos generada por el sistema r-t-q (Giardina et $a l ., 2000)$. Existen numerosas evidencias de que diferentes grupos indígenas de diversas regiones geográficas tienen sus propios métodos de manejo y aprovechamiento de los recursos naturales que resultaban de facto o deliberadamente en estrategias de conservación (Gómez-Pompa y Kaus, 1999; van der Wal, 1999). Sin embargo, al igual que en la región de estudio, es de esperar que estos mecanismos se están debilitando en la medida en que la intensidad y la frecuencia de uso de las parcelas se está incrementando.

Sustentabilidad del sistema roza-tumba-quema. El sistema agrícola r-t-q fue en principio ambientalmente sustentable debido a que en su forma tradicional no utilizaba agroquímicos (fertilizantes y pesticidas; Carter, 1989; Kleinman et al., 1995; Giardina et al., 2000), evitaba la erosión del suelo y aumentaba su fertilidad de manera natural mediante el desarrollo de vegetación secundaria (Dockersmith et al., 1999; Mertz y Magid, 2001). Mediante la quema se incrementa la disponibilidad de nutrientes para la cosecha (Giardina et al., 2000; Wan et al., 2001). El mantenimiento de este sistema tradicional promovía el uso de numerosos recursos, al integrar la producción de los cultivos y la obtención de productos asociados a la milpa, resaltando el aspecto sustentable del sistema (Carter, 1989; Toledo et al., 2003; Diemont et al., 2006). En este trabajo se encontró que la mayoría de las parcelas estudiadas aún se mantienen bajo el sistema tradicional de r-t-q en el que los períodos de descanso son el principal mecanismo para reestablecer la fertilidad y productividad del suelo. Sin embargo, el que cerca de $90 \%$ de las parcelas hayan presentado periodos de descanso máximos de siete años es una muestra del incremento de la frecuencia e intensidad de uso, lo que junto con la incorporación del uso de agroquímicos y el incremento de la frecuencia de las quemas en las parcelas, debilita la sustentabilidad de este sistema. Por otra parte, una cuarta parte de los productores alternó la milpa con pastizales para la ganadería, lo que indica que los campesinos ven en este uso del suelo una alternativa más atractiva y de mayor ingreso (Feder et al., 1985), con la consecuente pérdida del sistema tradicional de r-t-q, lo que elimina esta forma de producción ancestral que mantenía un nivel de resiliencia del sistema forestal (Gómez-Pompa y Kaus, 1999).

Intensidad y frecuencia de uso de las parcelas. La historia de uso de las parcelas en el área de estudio indica que en los últimos 40 años ha habido una reducción de los períodos de descanso, los cuales actualmente varían de 1 a 7 años (mediana de 4 años), evidenciando un proceso de intensificación que rompe con el esquema de descansos del sistema de r-t-q tradicional que permitía períodos de descanso de 15 a 20 años (Toledo, 1995; Toledo et al., 1995; Levy-Tacher, 2000). La intensificación del sistema de r-t-q -en términos de acortamiento de los períodos de descanso- no permite que el suelo recupere su fertilidad natural (Kauffman et al., 1993) ni que los acahuales alcancen suficiente complejidad estructural para crear condiciones microclimáticas de humedad y sombra. En este sentido, se encontró que los acahuales de poca edad ( 7 años) sopor tan un menor número de especies leñosas nativas propias del bosque maduro, perdiendo con ello la sustentabilidad del sistema en cuanto a su capacidad de conservar los recursos naturales (figura 5, cuadrante inferior izquierdo).

El incremento de la frecuencia de uso de las parcelas implica quemas también más frecuentes sobre poca biomasa acumulada, por lo que la incorporación de nutrientes al suelo queda restringida. Por otra parte, el fuego puede alcanzar temperaturas mayores que $200^{\circ} \mathrm{C}$ a niveles superficiales del suelo (Kauffman et al., 1993; Giardina et al., 2000), por lo que el incremento de su frecuencia puede estar destruyendo el banco de semillas, afectar la fauna del suelo, acelerar los procesos de erosión y afectar la dinámica de la sucesión (Quintana-Ascencio et al., 1996). La severidad del fuego resulta en diferencias dramáticas en la supervivencia de las plantas y por lo tanto en la diversidad biológica (Kauffman et al., 1993). Por otra parte, la probabilidad de expansión de fuego de la agricultura o los potreros es común en las áreas tropicales, afectando la dinámica 
de los remanentes de bosque, ya que los árboles tropicales no son resistentes al fuego, e inclusive los fuegos de baja intensidad pueden ser letales para mu chas especies arbóreas (Wan et al., 2001; Alvarado et al., 2004). Por ello, además de los procesos naturales de sucesión determinados por la edad del acahual, puede haber una fuerte influencia de la historia de uso de la parcela, como ha sido reportado para otras áreas tropicales (Zimmerman et al., 1995; van der Wal, 1999; Peña-Claros, 2001; Chinea, 2002; Thompson et al., 2002; Chinea y Helmer, 2003).

Sin duda, los procesos de deforestación e intensificación de uso de las parcelas son resultado de factores sociales, económicos y culturales (Fox et al., 2000; Ochoa-Gaona y González-Espinosa, 2000; Ochoa-Gaona, 2001). Por ejemplo, gran parte de los acahuales de mayor edad se encontraron en el Parque Nacional Palenque, debido a que en esta área hubo menor presión socioeconómica sobre los recursos naturales. Por otra parte, la expansión de los pastizales inducidos reduce las áreas forestales, lo que a su vez restringe la lluvia de semillas, provocando en última instancia un empobrecimiento de la diversidad de especies en las regiones neotropicales (Quintana-Ascencio et al., 1996). Otra limitante que influye en el acortamiento de los periodos de descanso es la baja disponibilidad de tierra de los productores (Fox et al., 2000; Ochoa-Gaona y GonzálezEspinosa, 2000). En este sentido, el proceso de empobrecimiento de la flora leñosa de los acahuales debe verse como un fenómeno multicausal, ya que de manera subyacente existen factores productivos, ambientales, sociales, económicos y culturales que lo explican y que aún es necesario abordar y entender de manera más precisa.

Conclusiones. Los resultados indican que alrededor de $50 \%$ de las especies leñosas registradas en los acahuales corresponden a especies propias de bosque maduro. Esto confirma la hipótesis de que la vegetación secundaria derivada de los sistemas de r-t-q con intensidad y frecuencia de uso bajas, amortigua el efecto de la fragmentación dentro de un mosaico de áreas agrícolas, acahuales, pastizales y remanentes de bosque. Sin embargo, el sistema de r-t-q muestra tendencias de mayor intensidad y frecuencia de uso, lo que se reflejará en matrices más homogéneas y de mayor contraste con las áreas forestales, impidiendo la dispersión, el establecimiento y el mantenimiento de la fracción de flora nativa que persiste en estos hábitats. Esto, a su vez, se reflejará en la alteración de los procesos de sucesión secundaria, comprometiendo la regeneración del bosque maduro y de las especies asociadas al mismo (QuintanaAscencio et al., 1996; Moya-García et al., 2003).

\section{Agradecimientos}

Agradecemos a Gilberto González Estrada, Isaías Martínez T., David Torres Zetina y José Luis Barragán Carrasco por su apoyo y orientación, a Miguel Martínez Icó, Nelly Jiménez Pérez y Henry Castañeda Ocaña por la identificación de las especies, a Christian Kampichler por la asesoría estadística y a Ramón Cuevas Guzmán por su asesoría en el análisis de ordenación, a Julia Carabias, Jorge Meave y a un revisor anónimo por sus valiosos comentarios. El Consejo Nacional de Ciencia y Tecnología (CONACYT) otorgó la beca 162872 a FHV y apoyó a través del proyecto Ecological and socioeconomic assessment of Landuse/Land-cover change in the humid tropics of eastern Tabasco and Selva Lacandona, Chiapas. Agradecemos a El Colegio de la Frontera Sur y al Instituto Manantlán de Ecología y Conservación de la Biodiversidad. Los ejidatarios de El Naranjo y Río Chancalá permitieron el acceso a sus parcelas.

\section{Literatura citada}

Alemán-Santillán T. 1989. Los sistemas de producción forestal y agrícola de roza. En: Parra-Vázquez M.R. Ed. El Subdesarrollo Agrícola en Los Altos de Chiapas, pp. 83-151. Universidad Autónoma Chapingo, Chapingo, Edo. de México.

Alvarado E., Sandberg D.V., de Carvalho J.A. Jr., Gielow R. y Santos J.C. 2004. Landscape fragmentation and fire vulnerability in primary forest adjacent to recent land clearings in the Amazon arc of deforestation. Floresta 34:169-174.

Bierregaard R.O. Jr., Lovejoy T.E., Kapos V., dos Santos A.A. y Hutchings R.W. 1992. The biological dynamics of tropical rainforest fragments. BioScience 42:859-866.

Brown S. y Lugo A.E. 1990. Tropical secondary forests. Journal of Tropical Ecology 6:1-32.

Carnevale N.J. y Montagnini F. 2002. Facilitating regeneration of secondary forests with the use of mixed and pure plantations of indigenous tree species. Forest Ecology and Management 163:217-227.

Carr D.L. 2004. Proximate population factors and deforestation in tropical agricultural frontiers. Population and Environment 25:585-612.

Carter H.O. 1989. Agricultural sustainability: an overview and research assessment. California Agriculture 43:16-18.

Chinea J.D. 2002. Tropical forest succession on abandoned farms in the Humacao Municipality of eastern Puerto Rico. Forest Ecology and Management 167:195-207.

Chinea J.D. y Helmer E.H. 2003. Diversity and composition of tropical secondary forests recovering from large-scale clearing: results from the 1990 inventory in Puerto Rico. Forest Ecology and Management 180:227-240.

De Jong B.H.J. y Montoya-Gómez G. 1994. Sustainable management of forest resources: a proposal for the highlands of Chiapas, México. En: Proceedings of the 1994 Symposium on Systems Analysis in Forest Resources. Management Systems for a Global Economy with Global Resources Concerns, pp. 7-17, septiembre 5-6. Pacific Grove, California.

De Jong B.H.J., Ochoa-Gaona S., Castillo-Santiago M.A., Ramírez-Marcial N. y Cairns M.A. 2000. Carbon flux patterns of land use/land cover change in the Selva Lacandona, Mexico. Ambio 29:504-511.

De Jong B.H.J., Ochoa-Gaona S., Rostrand J.D. y Bratckévich I. 
1990. Explicación de la terminología dendrológica y botánica En: De Jong B.H.J. Ed. Apuntes de Dendrología. Parte 1, pp. 117. Universidad Nacional Agraria. Managua.

De Vos J. 1992. Una selva herida de muerte, historia reciente de la Selva Lacandona. En: Vázquez-Sánchez M.A. y Ramos M.A. Eds. Reserva de la Biosfera Montes Azules, Selva Lacandona: Investigación para su Conservación, pp. 267-286. Centro de Estudios para la Conservación de los Recursos Naturales, A. C. Publicaciones Especiales de Ecosfera 1, San Cristóbal de las Casas, Chiapas.

Diemont S.A.W., Martin J.F. y Levy-Tacher S.I. 2006. Emergy evaluation of Lacandon Maya indigenous swidden agroforestry in Chiapas, Mexico. Agroforestry Systems 66:23-42.

Dirzo R. y García M.C. 1992. Rates of deforestation in Los Tuxtlas, a Neotropical area in Southeast Mexico. Conservation Biology 6:84-90.

Dockersmith I.C., Giardina C.P. y Sandford R.L. Jr. 1999. Persistence of tree related patterns in soil nutrients following slash-and-bum disturbance in the tropics. Plant and Soil 209:137-157.

Emrich A., Pokorny B. y Sepp C. 2000. La importancia del manejo de los bosques secundarios para la política de desarrollo. TÖB Series No. FTWF-18s, GTZ, Eschborn, Alemania.

Fahrig L. y Merriam G. 1985. Habitat patch connectivity and population survival. Ecology 66:1762-1768.

Feder G., Just R.E. y Zilberman D. 1985. Adoption of agricultural innovations in developing countries: a survey. Economic Development and Cultural Change 33:255-298.

Fox J., Truong D.M., Rambo A.T., Tuyen N. P., Cuc L.T. y Leisz S. 2000. Shifting cultivation: a new old paradigm for managing tropical forests. BioScience 50:521-528.

Freemark K.E., Boutin C. y Keddy C.J. 2002. Importance of farmland habitats for conservation of plant species. Conservation Biology 16:399-412.

García-Gil J.G. y Lugo-Hupb J. 1992. Las formas del relieve y los tipos de vegetación en la Selva Lacandona. En VázquezSánchez M.A. y Ramos M.A. Eds. Reserva de la Biosfera Montes Azules, Selva Lacandona: Investigación para su conservación, pp. 39-49. Centro de Estudios para la Conservación de los Recursos Naturales, A.C. Publicaciones Especiales de Ecosfera 1, San Cristóbal de las Casas, Chiapas.

Giardina C.P., Sanford R.L. Jr., Døckersmith I.C. y Jaramillo V.J. 2000. The effects of slash burning on ecosystem nutrients during the land preparation phase of shifting cultivation. Plant and Soil 220:247-260.

Gómez-Pompa A. y Kaus A. 1999. From pre-hispanic to future conservation alternatives: lessons from Mexico. Proceedings of the National Academy of Sciences of the United States of America 96:5982-5986.

Gómez-Pompa A. y Vázquez-Yanes C. 1976. Estudios sobre sucesión secundaria en los trópicos cálido-húmedos: el ciclo de vida de las especies secundarias. En Gómez-Pompa A., VázquezYanes C., del Amo S. y Butanda A. Eds. Regeneración de Selvas, pp. 559-592. Continental, México, D.F.

Gómez-Pompa A. y Vázquez-Yanes C. 1985. Estudios sobre la regeneración de selvas en regiones cálido-húmedas de México. En Gómez-Pompa A. y del Amo-R. S. Eds. Investigaciones sobre la Regeneración de Selvas Altas en Veracruz, México, Volumen II, pp. 1-25. Instituto Nacional de Investigaciones sobre Recursos Bióticos. Xalapa, Veracruz.
Guariguata M.R. y Ostertag R. 2001. Neotropical secondary forest succession: changes in structural and functional characteristics. Forest Ecology and Management 148:185-206.

Hernández-X., E. 1959. La agricultura. En: Beltrán E. Ed. Los Recursos Naturales Renovables del Sureste y su Aprovechamiento. Vol. 3, pp. 3-57. Instituto Mexicano de Recursos Naturales Renovables, A.C., México, D.F.

INE [Instituto Nacional de Ecología]. 2000. Programa de manejo de la Reserva de la Biosfera Montes Azules, México. Instituto Nacional de Ecología. México, D.F. <www.paot.org.mx/centro/ine-semarnat/anp/AN29.pdf>

ITTO [International Tropical Timber Organization]. 2002. Guidelines for restoration, management and rehabilitation of degraded and secondary tropical forests. ITTO Policy Development Series No 13, International Tropical Timber Organization, Yokohama.

Janzen D.H. 1986. The future of tropical ecology. Annual Review of Ecology and Systematics 17:305-324.

Kauffman J.B., Sanford R.L. Jr., Cummings D.L., Salcedo I.H. y Sampaio E.V.S.B. 1993. Biomass and nutrient dynamics associated with slash fires in neotropical dry forests. Ecology 74:140-151.

Kellman M. 1996. Redefining roles: plant community reorganization and species preservation in fragmented systems. Global Ecology and Biogeography Letters 5:111-116.

Kleinman P.J.A., Pimentel D. y Bryant R.B. 1995. The ecological sustainability of slash and burn agriculture. Agriculture, Ecosystems and Environment 52:235-249.

Kwesiga F. 1994. Rotational agrofore stry systems: ICRAF Training Course on Agroforestry Research for Development. Lecture handout - module II. Nairobi.

Lambin E.F., Turner B.L., Geist H.J., Agbola S.B., Angelsen A., Bruce J.W., Coomes O., Dirzo R., Fischer G., Folke C., George P.S., Homewodd K., Imbernon J., Leemans R., Li X., Moran E.F., Mortimore M., Ramakrishnan P.S., Richards J.F., Skånes H., Steffen W., Stone G.D., Svedin U., Veldkamp T.A., Vogel C. y Xu J. 2001. The causes of land- use and land-cover change: moving beyond the myths. Global Environmental Change 11:261-269.

Levy-Tacher S.I. 2000. Sucesión causada por roza-tumba-quema en las selvas de Lacanha, Chiapas. Tesis doctoral, Colegio de Postgraduados, Montecillo, Edo. de México, 140 pp.

López-García J. 1999. Estudio dendrológico de las especies comunes de la Selva Lacandona, Chiapas. Tesis de licenciatura, Universidad Autónoma de Chapingo. Chapingo, Edo. de México, 372 pp.

Lovejoy T.E., Bierregaard R.O., Rankin J.M. y Schubart H.O.R. 1983. Ecological dynamics of tropical forest fragments. En: Sutton S.L., Whitmore T.C. y Chadwick A.C. Eds. Tropical Rain Forest Ecology and Management, pp. 377-384. Blackwell Scientific Publications. Oxford.

Mertz O. y Magid, J. 2001. Shifting cultivation as conservation farming for humid tropical areas. En: Proceedings of the First World Congress on Conservation Agriculture, Vol. 2, pp. 55-59, Octubre 1-5. Madrid.

Moya-García X., Caamal A., Ku-Ku B., Chan-Xool E., Armendáriz I., Flores J., Moguer J., Noh-Poot M., Rosales M. y Xool-Domínguez J. 2003. La agricultura campesina de los mayas en Yucatán. LEISA Revista de Agroecología 19:7-17.

O'Brien K.L. 1995. Deforestation and climate change in the Selva 
Lacandona of Chiapas, Mexico: some preliminary results. Norsk Geografisk Tidsskrift 49:105-122.

Ochoa-Gaona S. 2001. Traditional land-use systems and patterns of forest fragmentation in the highlands of Chiapas, Mexico. Environmental Management 27:571-586.

Ochoa-Gaona S. y González-Espinosa M. 2000. Land-use patterns and deforestation in the highlands of Chiapas, Mexico. Applied Geography 20:17-42.

Parra-Vázquez M.R. y Mera-Ovando L.M. 1989. La organización social para la producción. En: Parra-Vázquez M. R. Ed. El Subdesarrollo Agrícola en Los Altos de Chiapas, pp. 315-405. Universidad Autónoma Chapingo, Chapingo, Edo. de México.

Pennington T.D. y Sarukhán J. 1998. Árboles Tropicales de México. 2a. Edición. Instituto de Ecología, Universidad Nacional Autónoma de México y Fondo de Cultura Económica. México, D.F.

Peña-Claros M. 2001. Secondary forest succession: processes affecting the regeneration of Bolivian tree species. PROMAB. Scientific Series 3. Riberalta, Bolivia.

Pool-Novelo L. 1997. Intensificación de la agricultura tradicional y cambio de uso del suelo. En: Parra-Vázquez M.R. y DíazHernández B.M. Eds. Los Altos de Chiapas: Agricultura y Crisis Rural I. Los Recursos Naturales, pp. 1-22. El Colegio de la Frontera Sur. San Cristóbal de las Casas, Chiapas.

Pool-Novelo L. y Hernández-X. E. 1991. El sistema agrícola de roza-tumba-quema: fundamentos, funcionamiento y perspectivas. Memoria del Primer Seminario sobre Manejo de suelos Tropicales en Chiapas. Centro de Investigaciones Ecológicas del Sureste, Publicación Especial, 11 p., San Cristóbal de las Casas, Chiapas.

Quintana-Ascencio P. F., González-Espinosa M., RamírezMarcial N., Domínguez-Vázquez G. y Martínez-Icó M. 1996. Soil seed banks and regeneration of tropical rain forest from milpa fields at the Selva Lacandona, Chiapas, Mexico. Biotropica 28:192-209.

Ramírez-Marcial N., Ochoa-Gaona S. y De Jong B.H.J. 1996. Fragmentación forestal y la vegetación de la Selva Lacandona, Chiapas, México. ECOSUR. Informe Técnico para la SEMARNAP. San Cristóbal de Las Casas, Chiapas, pp. 19 [Inédito].

Rzedowski J. 1978. Vegetación de México. Limusa, México, D.F.

Sader S.A., Sever T., Smoot J.C. y Richards M. 1994. Forest change estimates for the northern Petén region of Guatemala 19861990. Human Ecology 22:317-319.

Smith J., Sabogal C., De Jong W. y Kaimowitz D. 1997. Bosques secundarios como recurso para el desarrollo rural y la conservación ambiental en los trópicos de América Latina. CIFOR Occasional Paper No. 13. Center for International Forestry Research. Bogor, Indonesia.

Ter Braak C.J.F. y Smilauer P. 1998. CANOCO Reference Manual and User's Guide to CANOCO for Windows: Software for
Canonical Community Ordination (version 4). Microcomputer Power, Ithaca, Nueva York.

Thompson J., Brokaw N., Zimmerman J.K., Waide R.B., Everham E.M. III, Lodge D.J., Taylor C.M., Montiel D.G. y Fluet M. 2002. Land use history, environment, and tree composition in a tropical forest. Ecological Applications 12:1344 -1363.

Toledo V.M. 1995. Campesinidad, agroindustrialidad, sostenibilidad: los fundamentos ecológicos e históricos del desarrollo rural. Grupo Interamericano para el Desarrollo Sostenible de la Agricultura y los Recursos Naturales. Cuadernos de Trabajo 3:1-45

Toledo V.M., Batis A., Becerra R., Martínez E. y Ramos C.H. 1995. La selva útil: etnobotánica cuantitativa de los grupos indígenas del trópico húmedo de México. Interciencia 20:177187.

Toledo V.M., Ortiz-Espejel B., Cortés L., Moguel P. y Ordóñez M.D.J. 2003. The multiple use of tropical forest by indigenous people in Mexico: a case of adaptive management. Conservation Ecology 7:9. En línea:

<edcintl.cr.usgs.gov/SEMSOC/uploads/documents/carbon _in _forests/mutliple_use_of_tropical_forests-_ adapative_management.pdf>

Tucker J.M., Brondizio E.S. y Morán E.F. 1998. Rates of forest regrowth in eastern Amazonia: a comparison of Altamira and Bragantina regions, Pará state, Brazil. Interciencia 23:64-73.

Uhl C., Clark K., Clark H. y Murphy P. 1981. Early plant succession after cutting and burning in the Upper Rio Negro Region of the Amazon Basin. Journal of Ecology 69:631-649.

Uhl C. 1987. Factors controlling succession following slash-andburn agriculture in Amazonia. Journal of Ecology 75: 377-407. van der Wal, H. 1999. Chinantec Shifting Cultivation: Interactive Land Use. A Case-Study in the Chinantla, México, on Secondary Vegetation, Soils and Crop Performance under Indigenous Shifting Cultivation. Treemail Publishers, Heelsum, Holanda.

Vázquez-Yanes C. y Guevara-Sada S. 1985. Caracterización de los grupos ecológicos de árboles de la selva húmeda. En Gómez-Pompa A. y del Amo-R. S. Eds. Investigaciones Sobre la Regeneración de Selvas Altas en Veracruz, México. Volumen II, pp. 1-25. Instituto Nacional de Investigaciones sobre Recursos Bióticos. Xalapa, Veracruz.

Wan S., Hui D. y Luo Y. 2001. Fire effects on nitrogen pools and dynamics in terrestrial ecosystems: a meta-analysis. Ecological Applications 11:1349-1365.

Zimmerman J.K., Aide T.M., Rosario M., Serrano M. y Herrera L. 1995. Effects of land management and composition in the Luquillo Experimental Forest, Puerto Rico. Forest Ecology and Management 77: 65-76. 
Apéndice 1. Listado de especies con su acrónimo, familia y fase de desarrollo en que se registró en el campo. TE = Tipo de especie: * especies propias de bosque maduro. $\mathrm{A}=$ adulto, $\mathrm{J}=$ juvenil, $\mathrm{P}$ = plántula.

\begin{tabular}{|c|c|c|c|c|c|c|}
\hline Acrónimo & Especie1 & Familia & TE & A & $J$ & $\mathrm{P}$ \\
\hline Acco & Acacia cornigera (L.) Willd. & Leguminosae & & & 1 & \\
\hline Acglo & Acacia glomerosa Benth. & Leguminosae & * & 1 & 1 & 1 \\
\hline Acsp & Acacia sp. & Leguminosae & & 1 & 1 & 1 \\
\hline Acdi & Acalypha diversifolia Jacq. & Euphorbiaceae & & 1 & 1 & 1 \\
\hline Aсра & Acosmium panamense (Benth.) Yakovlev & Leguminosae & * & & 1 & \\
\hline Adsp & Adelia sp. & Euphorbiaceae & & 1 & 1 & 1 \\
\hline Aemo & Aegiphila monstrosa Moldenke & Verbenaceae & & 1 & 1 & 1 \\
\hline Alle & Albizia leucocalyx (Britton et Rose) L.Rico & Leguminosae & * & 1 & 1 & 1 \\
\hline Alla & Alchornea latifolia Sw. & Euphorbiaceae & * & 1 & 1 & \\
\hline Alyu & Alseis yucatanensis Standl. & Rubiaceae & * & & 1 & 1 \\
\hline Arco & Artocarpus communis J.R.Forst. et G.Forst. & Moraceae & $*$ & 1 & & \\
\hline Asgr & Astronium graveolens Jacq. & Anacardiaceae & * & 1 & 1 & 1 \\
\hline Blgu & Blepharidium guatemalense Standl. & Rubiaceae & * & & 1 & \\
\hline Bral & Brosimum alicastrum Sw. & Moraceae & * & & & 1 \\
\hline Bubu & Bucida buceras L. & Combretaceae & * & 1 & 1 & \\
\hline Busi & Bursera simaruba (L.) Sarg. & Burseraceae & * & 1 & 1 & 1 \\
\hline Bycr & Byrsonima crassifolia (L.) Kunth & Malpighiaceae & & & 1 & 1 \\
\hline Cabr & Calophyllum brasiliense Camb. & Guttiferae & $*$ & 1 & 1 & 1 \\
\hline Casp & Casearia sp. & Flacourtiaceae & & & 1 & \\
\hline Casy & Casearia sylvestris Sw. & Flacourtiaceae & & & 1 & \\
\hline Ceob & Cecropia obtusifolia Bertol. & Cecropiaceae & & 1 & 1 & \\
\hline Ceod & Cedrela odorata L. & Meliaceae & & 1 & 1 & 1 \\
\hline Cepe & Ceiba pentandra (L.) Gaertn. & Bombacaceae & * & 1 & & \\
\hline Ceno & Cestrum nocturnum $\mathrm{L}$. & Solanaceae & & & 1 & 1 \\
\hline $\mathrm{Ci01}$ & Citrus aurantifolia (Christm.) Swingle & Rutaceae & & 1 & 1 & \\
\hline $\mathrm{CiO} 2$ & Citrus aurantium L. & Rutaceae & & 1 & 1 & 1 \\
\hline Coba & Coccoloba barbadensis Jacq. & Polygonaceae & & 1 & 1 & 1 \\
\hline Cofl & Coccoloba floribunda (Benth.) Lindau & Polygonaceae & & & 1 & \\
\hline Covi & Cochlospermum vitifolium (Willd.) Spreng. & Cochlospermaceae & & 1 & 1 & 1 \\
\hline Coar & Cojoba arborea (L.) Britton et Rose & Leguminosae & * & & 1 & \\
\hline Coal & Cordia alliodora (Ruiz et Pav.) Oken & Boraginaceae & & 1 & 1 & 1 \\
\hline Cosp & Cordia sp. & Boraginaceae & & & 1 & \\
\hline Cude & Cupania dentata DC. & Sapindaceae & * & 1 & 1 & 1 \\
\hline Cuma & Cupania macrophylla Mart. & Sapindaceae & * & 1 & 1 & 1 \\
\hline Cype & Cymbopetalum penduliflorum (Dunal) Baill. & Annonaceae & * & & 1 & \\
\hline Chme & Chrysophyllum mexicanum Brandegee ex Standl. & Sapotaceae & * & 1 & 1 & 1 \\
\hline Dear & Dendropanax arboreus (L.) Decne. et Planch. & Araliaceae & * & 1 & 1 & 1 \\
\hline Digu & Dialium guianense (Aubl.) Sandwith & Leguminosae & * & & & 1 \\
\hline Diosp & Diospyros sp. & Ebenaceae & * & & 1 & \\
\hline Disp & Diphysa sp. & Leguminosae & & & 1 & \\
\hline Erfo & Erythrina folkersii Krukoff et Moldenke & Leguminosae & * & 1 & 1 & \\
\hline Esbe & Esenbeckia berlandieri Baill. ex Hemsl. & Rutaceae & & & & 1 \\
\hline Euac & Eugenia aff. acapulcensis Steud. & Myrtaceae & $*$ & & 1 & 1 \\
\hline Euca & Eugenia capuli (Schltdl. et Cham.) Hook. et Arn. & Myrtaceae & * & & 1 & \\
\hline Eusp & Eupatorium sp. & Compositae & & & 1 & \\
\hline Fiya & Ficus yaponensis Desv. & Moraceae & $*$ & 1 & 1 & \\
\hline Glse & Gliricidia sepium (Jacq.) Kunth ex Walp. & Leguminosae & & 1 & & \\
\hline Guul & Guazuma ulmifolia Lam. & Sterculiaceae & & 1 & 1 & \\
\hline Guco & Guettarda combsii Urb. & Rubiaceae & & 1 & 1 & \\
\hline Gusp & Guettarda sp. & Rubiaceae & & 1 & 1 & \\
\hline Hanu & Hampea nutricia Fryxell & Malvaceae & * & 1 & 1 & 1 \\
\hline Hedo & Heliocarpus donnell-smithii Rose & Tiliaceae & & 1 & 1 & 1 \\
\hline Hepa & Heliocarpus popayanensis Kunth & Tiliaceae & & 1 & 1 & 1 \\
\hline Hosp & Hoffmannia sp. & Rubiaceae & & & 1 & \\
\hline Inpa & Inga pavoniana G.Don & Leguminosae & * & 1 & & \\
\hline Inpu & Inga punctata Willd. & Leguminosae & * & 1 & 1 & 1 \\
\hline Jado & Jacaratia dolichaula (Donn. Sm.) Woodson & Caricaceae & & 1 & 1 & \\
\hline Limi & Lippia myriocephala Schltdl. et Cham. & Verbenaceae & & 1 & 1 & 1 \\
\hline
\end{tabular}

${ }^{1}$ La nomenclatura de las especies se basó en el sitio del Missouri Botanical Garden - w³tropicos. <mobot.mobot.org/W3T/Search/vast.html> 


\begin{tabular}{|c|c|c|c|c|c|c|}
\hline Acrónimo & Especie $^{1}$ & Familia & TE & A & $\mathbf{J}$ & $\mathbf{P}$ \\
\hline Loca & Lonchocarpus castilloi Standl. & Leguminosae & * & 1 & 1 & \\
\hline Made & Malmea depressa (Baill.) R.E.Fr. & Annonaceae & $*$ & & 1 & \\
\hline Magl & Malpighia glabra L. & Malpighiaceae & $*$ & 1 & 1 & 1 \\
\hline Main & Mangifera indica $\mathrm{L}$. & Anacardiaceae & & & 1 & \\
\hline Miim & Miconia impetiolaris (Sw.) D.Don ex DC. & Melastomataceae & $*$ & 1 & & \\
\hline Muca & Muntingia calabura L. & Elaeocarpaceae & & & 1 & \\
\hline Mylo & Myriocarpa longipes Liebm. & Urticaceae & $*$ & & 1 & 1 \\
\hline Mysp & Myriocarpa sp. & Urticaceae & & & 1 & \\
\hline Nele & Nectandra leucocome Rohwer & Lauraceae & $*$ & 1 & 1 & 1 \\
\hline Paaq & Pachira aquatica Aubl. & Bombacaceae & & 1 & 1 & \\
\hline Paac & Parmentiera aculeata (Kunth) Seem. & Bignoniaceae & & 1 & 1 & 1 \\
\hline Peam & Persea americana Mill. & Lauraceae & & 1 & 1 & \\
\hline Piaf & Picramnia aff. teapensis Tul. & Simaroubaceae & & & 1 & 1 \\
\hline Piae & Piper aeruginosibaccum Trel. & Piperaceae & & 1 & 1 & 1 \\
\hline Piau & Piper auritum Kunth & Piperaceae & & 1 & 1 & \\
\hline Pidi & Piper diandrum C.DC. & Piperaceae & & & 1 & \\
\hline Pisp & Piscidia sp. & Leguminosae & & & 1 & 1 \\
\hline Pitsp & Pithecellobium sp. & Leguminosae & $*$ & 1 & & \\
\hline Plyu & Platymiscium yucatanum Standl. & Leguminosae & $*$ & & 1 & 1 \\
\hline Plli & Pleuranthodendron lindenii (Turcz.) Sleumer & Flacourtiaceae & * & & 1 & \\
\hline Pola & Posoqueria latifolia (Rudge) Roem. et Schult. & Rubiaceae & * & & 1 & 1 \\
\hline Poar & Poulsenia armata (Miq.) Standl. & Moraceae & $*$ & & 1 & 1 \\
\hline Posa & Pouteria sapota (Jacq.) H.E.Moore et Stearn & Sapotaceae & * & & 1 & \\
\hline Posp & Pouteria sp. & Sapotaceae & $*$ & & & 1 \\
\hline Prco & Protium copal (Schltdl. et Cham.) Engl. & Burseraceae & $*$ & & & 1 \\
\hline Psox & Pseudolmedia oxyphyllaria Donn.Sm. & Moraceae & * & & 1 & 1 \\
\hline Psgu & Psidium guajava L. & Myrtaceae & & & 1 & \\
\hline Pssp & Psychotria sp. & Rubiaceae & & & 1 & 1 \\
\hline Ptro & Pterocarpus rohrii Vahl & Leguminosae & $*$ & 1 & 1 & \\
\hline Qufu & Quararibea funebris (La Llave) Vischer & Bombacaceae & $*$ & & & 1 \\
\hline Rigu & Rinorea guatemalensis (S.Watson) Bartlett & Violaceae & $*$ & & 1 & 1 \\
\hline Roji & Rollinia jimenezii Saff. & Annonaceae & $*$ & 1 & 1 & 1 \\
\hline Rogo & Rondeletia gonzaleoides Standl. & Rubiaceae & & 1 & 1 & \\
\hline Sasa & Sapindus saponaria L. & Sapindaceae & & 1 & 1 & \\
\hline Sama & Sapium macrocarpum Müll.Arg. & Euphorbiaceae & $*$ & 1 & 1 & 1 \\
\hline Sasp & Sapium sp. & Euphorbiaceae & $*$ & & 1 & \\
\hline Saya & Saurauia yasicae Loes. & Actinidiaceae & $*$ & 1 & 1 & \\
\hline Scpa & Schizolobium parahyba (Vell.) S.F.Blake & Leguminosae & & 1 & 1 & \\
\hline Sisa & Simira salvadorensis (Standl.) Steyerm. & Rubiaceae & $*$ & & 1 & \\
\hline Sian & Siparuna andina (Tul.) A.DC. & Monimiaceae & & & & 1 \\
\hline Spca & Spathodea campanulata P.Beauv. & Bignoniaceae & & & 1 & \\
\hline Spmo & Spondias mombin L. & Anacardiaceae & & 1 & 1 & 1 \\
\hline Stdo & Stemmadenia donnell-smithii (Rose) Woodson & Apocynaceae & $*$ & 1 & 1 & 1 \\
\hline Swcu & Swartzia cubensis (Britton et P.Wilson) Standl. & Leguminosae & $*$ & & 1 & 1 \\
\hline Swsp & Swartzia sp. & Leguminosae & $*$ & & 1 & \\
\hline Swma & Swietenia macrophylla King & Meliaceae & $*$ & & 1 & 1 \\
\hline Taro & Tabebuia rosea (Bertol.) A.DC. & Bignoniaceae & & & & 1 \\
\hline Team & Terminalia amazonia (J.F.Gmel.) Exell & Combretaceae & $*$ & & 1 & \\
\hline Trmi & Trema micrantha (L.) Blume & Ulmaceae & & 1 & 1 & 1 \\
\hline Trmo & Trichilia montana Kunth & Meliaceae & $*$ & & 1 & 1 \\
\hline Trme & Trichospermum mexicanum (DC.) Baill. & Tiliaceae & & 1 & 1 & \\
\hline Trra & Trophis racemosa (L.) Urb. & Moraceae & $*$ & 1 & 1 & 1 \\
\hline Valu & Vatairea lundellii (Standl.) Killip ex Record & Leguminosae & $*$ & 1 & 1 & 1 \\
\hline Zami & Zanthoxylum microcarpum Griseb. & Rutaceae & $*$ & & & 1 \\
\hline Zugu & Zuelania guidonia (Sw.) Britton et Millsp. & Flacourtiaceae & $*$ & 1 & & \\
\hline De01 & Desconocida 1 & Euphorbiaceae & & 1 & & \\
\hline De02 & Desconocida 2 & Leguminosae & & & 1 & \\
\hline De03 & Desconocida 3 & Solanaceae & & & 1 & \\
\hline De04 & Desconocida 4 & Solanaceae & & & 1 & \\
\hline De05 & Desconocida 5 & Desconocida & & 1 & & \\
\hline De06 & Desconocida 6 & Desconocida & & 1 & & \\
\hline
\end{tabular}

